\section{Paratropic Delocalized Ring Currents in Flattened Cyclooctatetraene Systems with Bond Alternation**}

Patrick W. Fowler, Remco W. A. Havenith, Leonardus W. Jenneskens, ${ }^{*}$ Alessandro Soncini, and Erich Steiner

The classical Hückel and London theories of monocyclic $\pi$ systems lead to an expectation of diatropic and paratropic ring currents in cycles with $4 n+2$ and $4 n \pi$ electrons, respectively. ${ }^{[1]}$ In practice, benzene famously bears out this expectation, ${ }^{[2,3]}$ but the $8 \pi$-antiaromatic cyclooctatetraene (COT) distorts to achieve a nonplanar closed-shell configuration with localized double bonds, ${ }^{[4]}$ and behaves chemically as a conjugated alkene..$^{[5]}$

Synthetic chemists have shown an interest in using annelation with rigid bridges to impose geometric constraints to localize the electronic structure of benzene ${ }^{[6-9]}$ and, conversely, to impose a delocalized structure on COT. ${ }^{[10,11]}$ The recent synthesis of a stable annelated cyclooctatetraene, tetrakis(bicyclo[2.1.1] hexeno)cyclooctatetraene (1, Scheme 1), ${ }^{[11]}$ in which the eight-membered ring is confined to the plane but retains strong bond alternation (X-ray average; $\Delta R(\mathbf{1})=$ $\left.0.169 \AA^{[11]}\right)$, raises the question of whether this molecule will now follow the original Hückel-London expectation and, in spite of its bond alternation, ${ }^{[12]}$ support a delocalized paratropic ring current. The positive nucleus-independent chemical shift (NICS) ${ }^{[13]}$ value of $\delta=10.6 \mathrm{ppm}$ calculated for the octagonal ring, though much smaller than that for planar COT $\left(2\left(D_{4 \mathrm{~h}}\right) \delta=27.2 \mathrm{ppm}\right),{ }^{[11]}$ is one indication of such a current. Unfortunately, $\mathbf{1}$ does not have the protons attached to its planar COT ring that would allow direct diagnosis by ${ }^{1} \mathrm{H}$ NMR spectroscopy, whilst its bridgehead protons lie outside the shielding/deshielding cone of the central ring. (In fact, the remoteness of the bridgehead protons explains the small size of the observed shift of $\delta=0.18 \mathrm{ppm},{ }^{[6,11]}$ despite reversal of ring currents, between $\mathbf{1}$ and tris(bicyclo[2.1.1]hexeno)benzene; the ${ }^{1} \mathrm{H}$ NMR chemical shifts of bridgehead protons are similar in several bicyclo[2.1.1]hexeno-clamped derivatives of benzene and COT. ${ }^{[6,11,14,15]}$ ).

Here we use computed current - density maps to answer the question of whether $\mathbf{1}$ supports a delocalized paratropic ring current. Consideration of the maps leads to a general analysis of the factors that decide when a constrained ring will carry a

[*] Prof. Dr. L. W. Jenneskens

Debye Institute, Department of Physical Organic Chemistry

Utrecht University

Padualaan 8, $3584 \mathrm{CH}$ Utrecht (The Netherlands)

Fax: (+31)30-253-4533

E-mail: jennesk@chem.uu.nl

Prof. Dr. P. W. Fowler, Dr. R. W. A. Havenith, A. Soncini,

Dr. E. Steiner

School of Chemistry, University of Exeter

Stocker Road, Exeter EX4 4QD (UK)

Fax: (+44)1392-26-3434

[**] We are grateful for an International Author Travel Grant from the Royal Society of Chemistry (Grant 0012289) to L.W.J. and thank the European Union TMR and Marie-Curie schemes (contracts FMRXCT097-0192 (Biofullerenes, R.W.A.H.) and HPMT-CT-2000-00016 (A.S.))

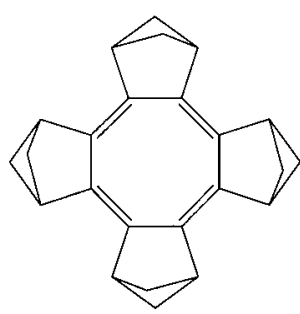

$1\left(D_{4 h}\right)$

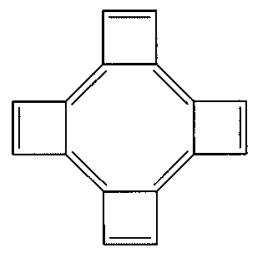

$4\left(D_{4 h}\right)$

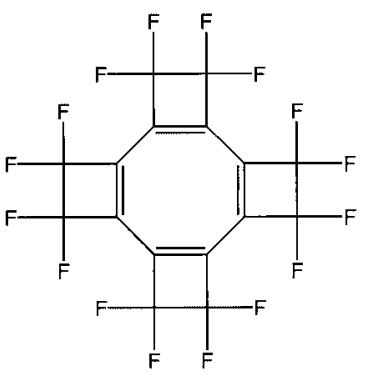

$6\left(D_{4 h}\right)$

Scheme 1. Molecules under study.

ring current. Comparisons of currents in $\mathbf{1}\left(D_{4 \mathrm{~h}}\right)$ with those in planar COT $\left(2\left(D_{4 h}\right)\right)$, COT in its tub-shaped equilibrium geometry $\left(\mathbf{3}\left(D_{2 \mathrm{~d}}\right)\right)$ and COT moieties in hypothetical tetrakis(cyclobutadieno)- $\left(\mathbf{4}\left(D_{4 \mathrm{~h}}\right)\right)$ and tetrakis(cyclobuteno)cyclooctatetraene $\left(\mathbf{5}\left(D_{4 \mathrm{~h}}\right)\right.$, Scheme 1$)$, will show that the choice of bridges used to effect clamping of COT into the plane determines whether or not a ring current exists.

Modern, distributed-origin, coupled Hartree-Fock methods such as the continuous transformation of origin of current density (CTOCD) method ${ }^{[16-19]}$ are particularly suited for the visualization and interpretation of ring currents ${ }^{[20]}$ (see Computational Section). Maps are shown in Figure 1 for the computed $\pi$-only and total $(\sigma+\pi)$ current densities in $\mathbf{1}\left(D_{4 \mathrm{~h}}\right)$. A strong, central paratropic ring current is evident in both a)

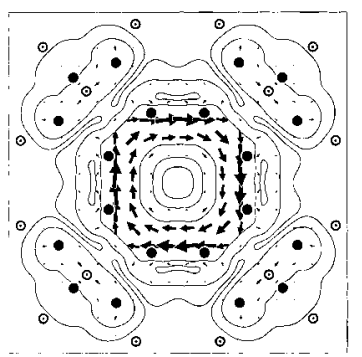

b)

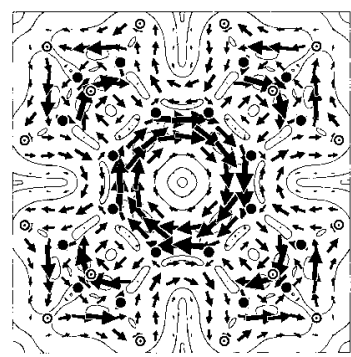

Figure 1. Maps of current density in tetrakis(bicyclo[2.1.1] hexeno)cyclooctatetraene (1): a) $\pi$-only, b) total $(\sigma+\pi)$ current ( carbon, $\odot$ hydrogen; nuclear positions projected in the plotting plane). 
maps. The $\pi$ contribution shows some variation in strength between alternate long and short bonds, which tends to equalize on inclusion of $\sigma$ effects. Apart from the main paratropic ring current, some extraneous features arise in the total $(\sigma+\pi)$ map where the plotting plane cuts through the bridge bonds of the four bicyclo[2.1.1] hexeno units. However, the constrained COT subunit in this new molecule does indeed sustain the classical Hückel $\pi$ current expected from its antiaromatic electron count. The question is whether this observation is a simple consequence of the enforced planarity of COT in $\mathbf{1}$.

The answer is provided by the planar form of COT itself. On optimization in $D_{4 \mathrm{~h}}$ symmetry, the resulting closed-shell COT structure $\left(2\left(D_{4 h}\right)\right)$, which is the transition state for tub-to-tub inversion, ${ }^{[21]}$ exhibits bond alternation similar to that in the subunit of the stable molecule $1\left(6-31 \mathrm{G}^{* *}: \Delta R(\mathbf{1})=0.184 \AA\right.$, $\Delta R(\mathbf{2})=0.153 \AA$ ). Planar COT (2) also displays the classical intense paratropic ring current expected for an antiaromatic $\pi$ system (Figure $2 \mathrm{a}$ ), associated here with the splitting into $\mathrm{b}_{2 \mathrm{u}}$ HOMO and $\mathrm{b}_{1 \mathrm{u}}$ LUMO in $D_{4 \mathrm{~h}}$ of the degenerate $\mathrm{e}_{2 \mathrm{u}}$ HOMO of the $D_{8 \mathrm{~h}}$ open-shell. ${ }^{[22]}$ The reduced ring current of the stable molecule $\mathbf{1}$ is consistent with its larger HOMO-LUMO gap (1: $9.4 \mathrm{eV}$ versus 2: $8.5 \mathrm{eV}$ ). a)

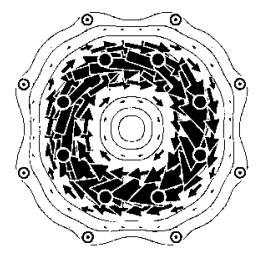

b)

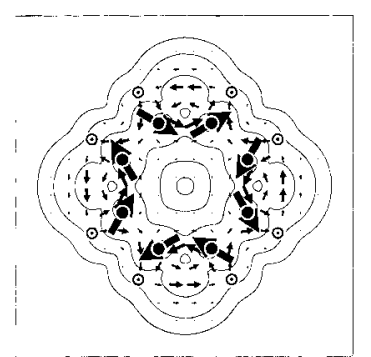

Figure 2. Maps of $\pi$-current density in a) planar COT (2), b) tub-shaped COT (3) $(\bullet$ carbon, $\odot$ hydrogen; in (a) nuclear positions projected in the plotting plane. In (b) the median plane was used as the plotting plane).

Relaxation of COT to its tub-shaped equilibrium geometry $3\left(D_{2 \mathrm{~d}}\right)$ has little effect on the bond alternation $\left(6-31 \mathrm{G}^{* *}\right.$ : $\Delta R(\mathbf{3})=0.155 \AA$ ) but destroys the delocalized ring current, as Figure $2 \mathrm{~b}$ shows. Long and short bond lengths are similar for all three COT structures (1, X-ray averages 1.331(1)/ 1.500(1) $\AA$; 6-31G** values: $\mathbf{1}\left(D_{4 \mathrm{~h}}\right), 1.323 / 1.507 \AA, \mathbf{2}\left(D_{4 \mathrm{~h}}\right)$, $\left.1.326 / 1.479 \AA, \mathbf{3}\left(D_{2 \mathrm{~d}}\right), 1.323 / 1.478 \AA\right)$. The $\pi$-only map for $\mathbf{3}$ drawn in the median plane of the molecule shows localized circulations from the four double bonds, two above and two below the plotting plane. From this comparison of $\mathbf{2}$ and $\mathbf{3}$, the importance of planarity is clear, notwithstanding previous literature statements, ${ }^{[12]}$ but planarity alone is insufficient to ensure presence of a current, as the next example shows. In 4 $\left(D_{4 \mathrm{~h}}\right.$; see Scheme 1) the COT subunit is again clamped in the plane, in this case by annelation with rigid cyclobutadieno rings. In contrast to $\mathbf{1}$, the $\pi$ and total $(\sigma+\pi)$ maps for $\mathbf{4}$ (Figure 3) show the typical signature of localized double bonds. No paratropic $\pi$ ring current is discernible in the COT unit of 4, though the unsaturated four-membered cycles themselves support central $(\sigma)$ paratropic contributions.

This quenching of the paratropic COT $\pi$ current in $\mathbf{4}$ arises specifically from the conjugation of the clamping group with a)

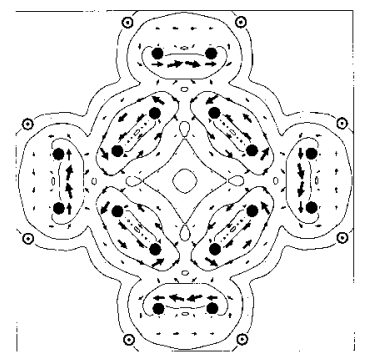

c)

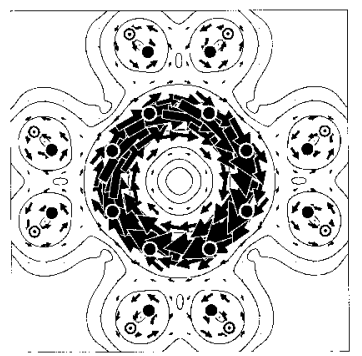

b)

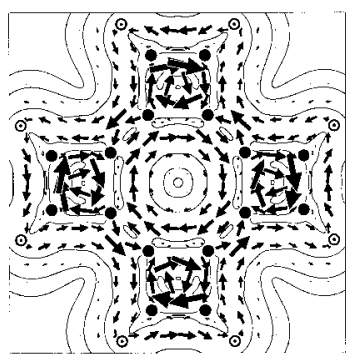

d)

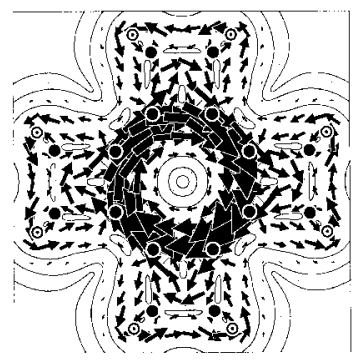

Figure 3. Maps of current density in tetrakis(cyclobutadieno)cyclooctatetraene (4) and tetrakis(cyclobuteno)cyclooctatetraene (5): a) $\pi$-only current in $\mathbf{4}, \mathrm{b}$ ) total $(\sigma+\pi)$ current in $\mathbf{4}, \mathrm{c}) \pi$-only current in $\mathbf{5}$, and d) total $(\sigma+\pi)$ current in $\mathbf{5}$ ( carbon, $\odot$ hydrogen; nuclear positions projected in the plotting plane)

the central ring. When the external $\pi$ bonds of $\mathbf{4}$ are hydrogenated, to give the planar tetrakis(cyclobuteno) derivative $5\left(D_{4 \mathrm{~h}}\right)$, the COT paratropic current is restored (Figure $3 \mathrm{c}$ ). The difference in current maps between $\mathbf{1}, \mathbf{5}$ on the one hand and $\mathbf{4}$ on the other is evidently not a consequence of differences in bond alternation, $\left(6-31 \mathrm{G}^{* *}: \Delta R(\mathbf{4})=1.501-\right.$ $1.317=0.184 \AA, \Delta R(\mathbf{5})=1.491-1.317=0.174 \AA)$. The explanation of the switch from localized to delocalized behavior lies instead in the difference between the $\pi$ electronic structures of the 16-electron conjugated system in $\mathbf{4}$ and the eightelectron COT system in $\mathbf{1}, \mathbf{2}$, and $\mathbf{5}$. As noted above, the planar COT ring has $a b_{2 u}$ HOMO and $b_{1 u}$ LUMO (Figure 4), and these are also HOMO and LUMO in $\mathbf{1}$ and $\mathbf{5}$. The $b_{2 u} \rightarrow b_{1 u}$ occupied-to-virtual transition has the symmetry of a rotation about the magnetic field direction, and therefore produces a paratropic contribution. ${ }^{[22]}$ This low-energy transition dominates the total $\pi$ ring current and determines its paratropic sense. In 4 however, the interaction of the central COT $\pi$ system (bonding orbitals $a_{2 u}, e_{g}, b_{2 u}$; antibonding $b_{1 u}, e_{g}, a_{1 u}$ ) with the four outer $\pi$ bonds (bonding combinations $a_{2 u}, e_{g}, b_{1 u}$; antibonding $b_{2 u}, e_{g}, a_{1 u}$ ) pushes the occupied $b_{2 u}$ orbital down, below $2 \mathrm{a}_{2 \mathrm{u}}(\mathrm{HOMO}-1)$ and $2 \mathrm{e}_{\mathrm{g}}(\mathrm{HOMO})$, and pushes the empty $b_{1 u}$ orbital up, above the $3 \mathrm{e}_{\mathrm{g}}$ (LUMO) and $1 \mathrm{a}_{1 \mathrm{u}}$ $(\mathrm{LUMO}+1)$ (Figure 4). The large paratropic $1 \mathrm{~b}_{2 \mathrm{u}}$ orbital contribution is thereby suppressed and replaced by a mixture of weaker contributions ( $2 \mathrm{e}_{\mathrm{g}} \rightarrow 3 \mathrm{e}_{\mathrm{g}}$ (paratropic), $2 \mathrm{a}_{2 \mathrm{u}} \rightarrow 3 \mathrm{e}_{\mathrm{g}}$ (diatropic), $1 b_{2 u} \rightarrow 3 e_{g}$ (diatropic)), which sum to localized diamagnetic circulations in the eight $\pi$ bonds of 4 . In the language of reference [22], two-electron paramagnetism in $\mathbf{1}$, $\mathbf{2}$, and $\mathbf{5}$ has been replaced by eight-electron diamagnetism in 4. This is a direct consequence of $\pi-\pi$ interaction between COT and the clamping groups (see also references $[23,24]$; if the exo single bonds of $\mathbf{4}$ are artificially stretched to $2 \AA$, for example, the central paratropic COT current reappears). 


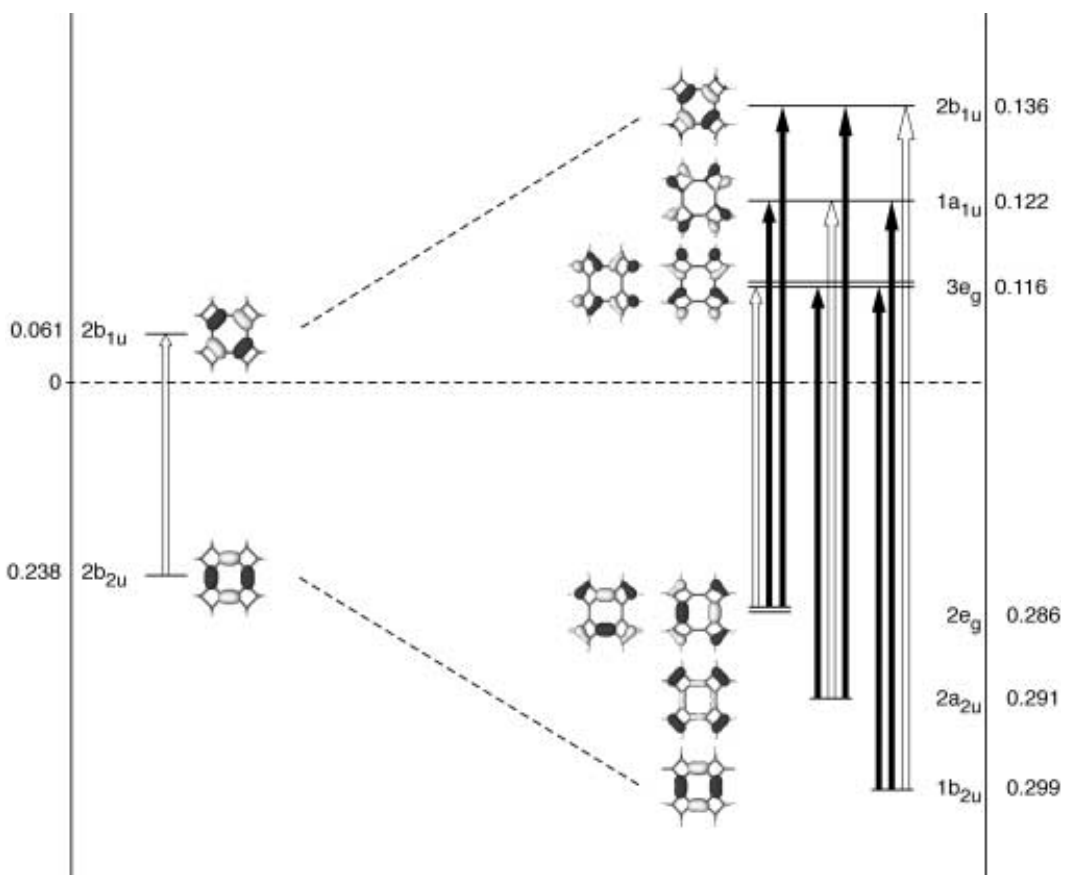

Figure 4. Three-dimensional contour maps of the HOMOs and LUMOs of 4 (right) and $\mathbf{5}$ (left), indicating their energies (Hartree) and symmetries. Black arrows represent translational transitions and white arrows rotational transitions from occupied levels. ${ }^{[2]}$ The $\pi$ molecular orbitals of $\mathbf{1}$ and $\mathbf{2}$ are similar to those of $\mathbf{5}$.

The orbital picture also gives a ready explanation of the properties of other species such as perfluorinated tetrakis(cyclobuteno)cyclooctatetraene $\left(\mathbf{6}\left(D_{4 \mathrm{~h}}\right)\right.$, Scheme 1$){ }^{[25]}$ Replacement of $\mathrm{H}$ by $\mathrm{F}$ in 5 stabilizes the endo tautomer $\mathbf{6}^{[12]}$ but as this corresponds to a simple switch of HOMO and LUMO symmetries, the paratropic ring current should be retained. Explicit CTOCD-DZ calculations of current maps (not shown) confirm this expectation: $\mathbf{6}$ has indeed a strong central paratropic $\pi$ current of similar intensity to that in $\mathbf{5}$.

The series of calculations described here show that when COT is made planar, its magnetic response is essentially independent of the degree of bond alternation. A paratropic $\pi$ ring current is compatible with clamping by groups that exert a purely geometrical effect. The crucial factor in the survival of the ring current is the rotationally allowed COT HOMOLUMO transition; when interaction with the $\pi$ system of an unsaturated clamping group opens up the gap between the $b_{2 u}$ and $b_{1 u}$ orbitals, the ring current is destroyed. In this context, the bicyclo[2.1.1] hexeno and cyclobuteno clamping groups are passive spectators, whereas the cyclobutadieno clamp actively suppresses the antiaromatic central current. The bicyclo[2.2.2] octene group ${ }^{[10]}$ is also a spectator in this sense to a diamagnetic current in $\mathrm{COT}^{2-}$.

A parallel distinction in localization of central current is expected to apply to the equivalent derivatives of benzene. ${ }^{[6-9,26]}$

\section{Computational Section}

Methods: The magnetic properties of all molecules were computed by using distributed-origin methods, ${ }^{[16,17]}$ at the coupled Hartree-Fock level, ${ }^{[18,19]}$ with the SYSMO program. ${ }^{[27]}$ For the current-density maps, well-converged results are given by the DZ (diamagnetic zero) variant of the CTOCD (continuous transformation of origin of current density) method, where the current density at any point in space is calculated with that point as origin. ${ }^{[16-19]}$ The method has the usual advantages of distributed-origin approaches: superior convergence with basis size and physically realistic current maps with modest basis sets (here $6-31 \mathrm{G}^{* *}$ ). Optimized geometries, confirmed as minima $(\mathbf{1}, \mathbf{3}-\mathbf{6})$ or in one case a transition state (2: one imaginary frequency), were computed in the same basis.

For all but 3 (see legend to Figure 2), current densities induced by a unit magnetic field acting along the principal axis are plotted in a plane $1 a_{0}$ above that of the central ring (that is, close to the maximum $\pi$ density and with current essentially parallel to the molecular plane, as shown previously ${ }^{[28]}$ ), the plotting area is $16 \times 16 a_{0}^{2}$, the contours denote the modulus of the current density, and the vectors represent in-plane projections of current. In all plots, diamagnetic circulation is shown anticlockwise and paramagnetic circulation clockwise.

Received: June 11, 2001 Revised: February 18, 2002 [Z17268]

[1] F. London, J. Phys. Radium 1937, 8, 397-409.

[2] L. Pauling, J. Chem. Phys. 1936, 4, 673-677.

[3] J. A. Pople, J. Chem. Phys. 1956, 24, 1111.

[4] J. March, Advanced Organic Chemistry, Wiley, New York 1986.

[5] For a general survey: M. Jones Jr., Organic Chemistry, Norton, New York, 2000.

[6] N. L. Frank, K. K. Baldridge, J. S. Siegel, J. Am. Chem. Soc. 1995, 117, 2102-2103.

[7] H.-B. Bürgi, K. K. Baldridge, K. Hardcastle, N. L. Frank, P. Gantzel, J. S. Siegel, J. Ziller, Angew. Chem. 1995, 107, 1575-1577; Angew. Chem. Int. Ed. Engl. 1995, 34, 1454-1456.

[8] R. Diercks, K. P. C. Vollhardt, J. Am. Chem. Soc. 1986, 108, $3150-$ 3152.

[9] D. Holmes, S. Kumaraswamy, A. J. Matzger, K. P. C. Vollhardt, Chem. Eur. J. 1999, 5, 3399-3412.

[10] K. Komatsu, T. Nishinaga, S. Aonuma, C. Hirosawa, K. Takeuchi, H. J. Lindner, J. Richter, Tetrahedron Lett. 1991, 32, 6767-6770.

[11] A. Matsuura, K. Komatsu, J. Am. Chem. Soc. 2001, 123, 1768-1769.

[12] K. K. Baldridge, J. S. Siegel, J. Am. Chem. Soc. 2001, 123, 1755-1759.

[13] P. von R. Schleyer, C. Maerker, A. Dransfeld, H. Jiao, N. J. R. van Eikema Hommes, J. Am. Chem. Soc. 1996, 118, 6317-6318.

[14] M. Pomerantz, R. N. Wilke, G. W. Gruber, U. Roy, J. Am. Chem. Soc. 1972, 94, 2752-2758.

[15] M. Christl, H. Reuchlein, Angew. Chem. 1990, 102, 1090-1091; Angew. Chem. Int. Ed. Engl. 1990, 29, 1035-1037.

[16] T. A. Keith, R. F. W. Bader, Chem. Phys. Lett. 1993, 210, 223-231.

[17] T. A. Keith, R. F. W. Bader, J. Chem. Phys. 1993, 99, 3669-3682.

[18] S. Coriani, P. Lazzeretti, M. Malagoli, R. Zanasi, Theor. Chim. Acta 1994, 89, $181-192$.

[19] R. Zanasi, J. Chem. Phys. 1996, 105, 1460-1469.

[20] E. Steiner, P. W. Fowler, L. W. Jenneskens, Angew. Chem. 2001, 113, 375-379; Angew. Chem. Int. Ed. 2001, 40, 362-366.

[21] J. L. Andrés, O. Castaño, A. Morreale, R. Palmeiro, R. J. Gomperts, J. Chem. Phys. 1998, 108, 203-207.

[22] E. Steiner, P. W. Fowler, J. Phys. Chem. A 2001, 105, 9553-9562; E. Steiner, P. W. Fowler, Chem. Commun. 2001, 2220-2221.

[23] R. Faust, E. D. Glendening, A. Streitwieser, K. P. Vollhardt, J. Am. Chem. Soc. 1992, 114, 8263-8268.

[24] K. K. Baldridge, J. S. Siegel, J. Am. Chem. Soc. 1992, 114, 9583 - 9587.

[25] F. W. B. Einstein, A. C. Willis, W. R. Cullen, R. L. Soulen, J. Chem. Soc. Chem. Commun. 1981, 526-528.

[26] P. W. Fowler, R. W. A. Havenith, L. W. Jenneskens, A. Soncini, E. Steiner, Chem. Commun. 2001, 2386-2387.

[27] P. Lazzeretti, R. Zanasi, SYSMO package (University of Modena), 1980. Additional routines for evaluation and plotting of current density, E. Steiner, P. W. Fowler, unpublished results.

[28] A. Soncini, P. W. Fowler, I. Černušak, E. Steiner, Phys. Chem. Chem. Phys. 2001, 3, 3920-3923, and references therein. 